\title{
A multidisciplinary chronic lung disease team in a neonatal intensive care unit is associated with increased survival to discharge of infants with tracheostomy
}

\author{
Taylor P. Hansen ${ }^{1} \cdot$ Janelle Noel-MacDonnell $\mathbb{D}^{2,3} \cdot$ Sara Kuckelman $^{4} \cdot$ Michael Norberg $^{1} \cdot$ William Truog $^{1,3} \cdot$ \\ Winston Manimtim (1) ${ }^{1,3}$
}

Received: 12 June 2020 / Revised: 20 November 2020 / Accepted: 27 January 2021 / Published online: 1 April 2021

(c) The Author(s), under exclusive licence to Springer Nature America, Inc. 2021

\begin{abstract}
Objective To determine if multidisciplinary team-based care of severe BPD/CLD infants improve survival to discharge. Design/methods Retrospective review of severe BPD/CLD infants cared for by dedicated multidisciplinary CLD team using consensus-driven protocols and guidelines.

Results Total of 267 patients. Median gestational age was 26 weeks (IQR 24, 32); median birth-weight was 0.85 (IQR 0.64, 1.5). Twenty-four percent were preterm with severe BPD, $46 \%$ had other primary respiratory diseases (none BPD diseases). Total number of patients, proportion of patients with tracheostomy, prematurity, and genetic diagnoses increased over time. $88.8 \%$ survived to discharge. Unadjusted logistic regression showed that tracheostomy was not associated with odds of death; secondary pulmonary hypertension was associated with odds of tracheostomy $(\mathrm{OR}=1.795 p$ value $=0.0264)$, or death $(\mathrm{OR}=8.587 p$ value $=<0.0001)$, or tracheostomy + death $(\mathrm{OR}=13.58 p$ value $=0.0007)$.

Conclusions Over time, mortality improved for infants with tracheostomy cared for by a multidisciplinary severe BPD/CLD team. Secondary pulmonary hypertension was associated with tracheostomy, or death, or tracheostomy + death.
\end{abstract}

\section{Introduction}

Bronchopulmonary dysplasia (BPD), first described by Northway in 1967, remains the most-common complication of premature birth $[1,2]$. Currently, the most accepted definition of BPD is the need for oxygen or respiratory support at 36 weeks' postmenstrual age (PMA). BPD is considered severe if the infant remains ventilator-dependent

Supplementary information The online version contains supplementary material available at https://doi.org/10.1038/s41372021-00974-2.

Winston Manimtim

wmmanimtim@cmh.edu

1 Division of Neonatology, Children's Mercy Kansas City, Kansas City, MO, USA

2 Health Services and Outcome Research, Children's Mercy Kansas City, Kansas City, MO, USA

3 School of Medicine, University of Missouri-Kanas City, Kansas City, MO, USA

4 School of Medicine, University of Kansas, Lawrence, KS, USA with $>30 \% \mathrm{FiO}_{2}$ requirement [3]. Patients with severe BPD often have severe complications, including pulmonary hypertension, poor growth, long-term pulmonary morbidity, and neurodevelopmental disability [4]. The term BPD is often used interchangeably with chronic lung disease of prematurity (CLDP). The American Thoracic Society (ATS) proposed that BPD and CLDP are both forms of chronic lung disease of infancy (CLDI), in addition to any pulmonary disorders that originated during the neonatal period [5].

There is a lack of published guidelines and national standards to treat infants with severe BPD/CLD. Practice variability has contributed to the challenges to prevent and improve outcomes of infants with severe BPD/CLD. Both inter-center and within center variability in the care of infants with severe BPD/CLD have been demonstrated for the use of multiple therapies such as bronchodilators, diuretics, and inhaled nitric oxide. A recently published multidisciplinary quality improvement (QI) initiative successfully reduced the rate of severe BPD in a level-IV neonatal intensive care unit (NICU) from 57 to $21 \%$ over 3 years [6]. With the recognition that CLD is a multifactorial disease, management guidelines are slowly emerging 
specifically for those with established severe phenotypes. In 2008 , it was observed that an increasing number of very complex CLD patients was being cared for in our regional referral level-IV intensive care nursery (ICN). These complex patients were requiring multi-specialty care that was being compromised by frequently rotating healthcare providers at all levels.

Parents were frustrated by the chronicity of patient care needs and dissatisfied with the inconsistencies of care plans provided to the patients. To address these concerns, a multidisciplinary task force recommended the formation of a dedicated multidisciplinary team with a limited number of attending neonatologists and support staff. Here, we outline the development, composition, and operations of our CLD team, the effect it has had in this patient population, the culture change observed, and the results we have found on survival to NICU discharge. The objective of this study is therefore, to describe the effect of a multidisciplinary teambased approach in the survival to NICU discharge of infants with severe BPD/CLD who received tracheostomy during their initial NICU hospitalization, and secondly, to describe patient characteristics and identify risk factors for death prior to hospital discharge.

\section{Methods}

\section{Development, goals, composition, and operations of the CLD multidisciplinary team}

The Children's Mercy Kansas City Intensive Care Nursery (ICN) is an 84-bed level-IV regional neonatal referral center staffed by 32 neonatologists, 7 neonatal fellows, and $\sim 75$ neonatal nurse practitioners. In January 2010, a dedicated multi-interdisciplinary CLD team was formed to provide family-centered care to infants with CLD. The team's vision was to improve the outcome of infants with chronic lung disease who do not respond to standard neonatal therapies. The team's goals were as follows: (a) to develop a multi- and interdisciplinary center of excellence for CLD patients within the NICU; (b) to standardize the approach to CLD care by applying an evidence-based medicine approach, and when evidence is lacking, to develop a consensus-driven clinical practice guideline; and (c) to pursue research questions related to the prevention and management of CLD.

The CLD team consists of dedicated neonatologists with a wide range of expertise in managing infants with severe CLD. The team is supported by a dedicated pediatric pulmonologist, a cardiologist, and otolaryngologists. The team also includes a team facilitator/coordinator, primary bedside NICU nurses, neonatal nurse practitioners, respiratory therapists, tracheostomy coordinator, clinical pharmacologist, dietician, social worker, psychologist, parent advisor, occupational, speech and language therapists, and members of the infant tracheostomy and home ventilator team, pulmonary hypertension team, and palliative care team. The group meets biweekly to discuss each individual case and to propose management for acute and long-term issues. These meetings also provide a regular venue for staff education, review of protocols, evaluation of consensus guidelines, develop and implement QI practices as well as generation of clinical research questions specific to patients with CLD.

\section{Identification of the CLD patient cohort}

At the center of the CLD team approach is the identification of the patient population served by the dedicated team. The population is comprised of patients broadly categorized with a diagnosis of CLDI based on the ATS definition [5]. They include, but are not limited to, the following: (a) infants who were born extremely premature and developed severe BPD, who remained oxygen- and ventilatordependent by 36 weeks' PMA, based on the National Institute of Child Health and Development (NICHD) criteria [4]; (b) infants with respiratory and airway anomalies, either congenital or acquired, who were likely to need tracheostomy; (c) infants with congenital heart disease with chronic congestive heart failure requiring positive pressure ventilatory support; (d) infants with neurologic conditions who were ventilator-dependent; and (3) infants with various genetic diagnoses associated with long-term respiratory support.

\section{Standardized protocols, practice guidelines, and staff educational presentations}

As the process of standardization has been shown to improve outcomes within healthcare settings, standard treatment protocols and guidelines have enabled consistency of care and reduction of practice variability among care providers, Over the years, the team has developed consistent evidence-based and consensus-driven approach protocols in the management of infants with severe CLD (Appendix 1). In addition, a quarterly educational presentation is given by one of the team members to provide updated information relevant to the management of infants with CLD. The topics of these presentations include ventilation strategies, use of various medications in CLD, and home environmental assessment. A complete list of all the presentations is provided in Appendix 2.

\section{QI initiatives}

To further promote consistency of practice, several QI projects specific to the CLD patient population were developed and implemented over the course of several 
years. Examples of these QI initiatives include the following: (a) tracheitis algorithm for infants with tracheostomy, led by our clinical pharmacologist (Appendix 3); (b) a metabolic bone health screening in infants on long-term furosemide therapy, done in collaboration with the metabolic bone specialists; (c) standardized parent/caregiver education and training and roadmap to home, led by our tracheostomy coordinator (Appendix 4); and (d) a familycentered care initiative.

\section{Study design}

The list of the entire patient cohort was provided by the CLD team coordinator and was part of the database of the Center for Infant Pulmonary Disorders (CIPD), located at Children's Mercy Kansas City. The CIPD houses deidentified data for this study with Children's Mercy Institutional Review Board approval. Included in the cohort were all infants cared for by the CLD multidisciplinary team members from the time the committee was formed in 2010 to December 31, 2018. Infants with the chronic lung disease from 2008 to 2009 served as historical baseline data prior to establishment of CLD team. Diagnoses were extracted by the ICD-9 and ICD-10 codes (The International Classification of Diseases, Ninth and Tenth Revisions) from the electronic medical records.

\section{Demographic and outcome data}

Demographic data included gestational age at birth, birth weight, sex, race, and chronologic age (and adjusted age for premature infants in PMA in weeks). Patients were considered in-born if they were delivered at Children's Mercy Fetal Health Center, Truman Medical Center or transferred within $48 \mathrm{~h}$ of birth. Almost all infants have multiple diagnoses on admission therefore, only the primary diagnosis was used to categorize the entire cohort into six major subgroups, namely: (a) primary respiratory (non-BPD) conditions, (b) prematurity with severe BPD, (c) genetic diagnosis, (d) cardiac anomalies, (e) neurologic conditions, and (f) others, for those who were not able to be categorized in any of the other five subgroups. The number of patients in each of these subgroups was tracked over time. Data on respiratory support at the time of admission and at discharge were also collected.

Information was collected on the presence of comorbid conditions (pulmonary hypertension, cardiac, neurologic, genetic, ophthalmic, infection, BPD, gastrointestinal, gastrostomy tube, and fundoplication) and use of medications specific to CLD (diuretics, bronchodilators, and steroids) during hospitalization until discharge. The need for tracheostomy, adjusted age at tracheostomy, need for home ventilation at the time of discharge, the age at discharge and the patients' residence zip codes at discharge were reported. All deaths that occurred during hospitalization in the entire cohort were reviewed, including autopsy results when available, and the causes of death were noted, including the age at death.

\section{Statistical analysis}

General descriptive statistics of patient characteristics were used. For continuous variables, independent T-test was used, while comparison between categories was done using the chi-square or Fisher's exact test. An unadjusted logistic regression analysis was conducted for ten different comorbidities to determine if any is associated with death prior to discharge, tracheostomy, and combined outcome of death and tracheostomy. In addition, the trend in the number of patients annually was analyzed to show whether there was a significant increase in the total number of patients, the number of patients for each specific diagnosis subgroup, the number of infants who had tracheostomy, and the number of patients who died before discharge, over the entire study period.

\section{Results}

\section{Description of the cohort}

A total of 267 patients were identified from 2008 to 2018 . Patients born from 2008 to $2009(n=15)$ with CLD served as a reference data point but not a comparison group prior to the establishment of the CLD multidisciplinary team in 2010. Of the entire cohort, $56 \%$ were male and the majority were Caucasians (62.2\%), followed by African Americans (22.8\%), Hispanics (6.7\%), Asians (0.7\%), and others (7.6\%). The median gestational age at birth was 26 weeks (IQR: 24, 32); mean (SD) was 28.5 weeks (5.2) weeks; the median birth-weight was $0.85 \mathrm{~kg}$ (IQR 0.6,1.5), and mean birth-weight was $1.3 \mathrm{~kg}(\mathrm{SD}=1.0)$ (Table 1).

Based on the primary diagnosis on admission, the proportions of patients by subgroups were primary respiratory (non-BPD) conditions (44\%) (patients with congenital diaphragmatic hernia, pulmonary hypoplasia, choanal atresia, subglottic stenosis, vocal cord paralysis, and surfactant protein deficiency, etc.), extremely preterm infants (born $<28$ weeks' gestation) with severe BPD (24\%), genetic conditions (14\%) (variety of genetic anomalies such as trisomies, Treacher Collins, Beckwith-Wiedemann, PraderWilli, Goltz, Beare-Stevenson, DiGeorge syndromes, etc.), cardiac anomalies (6\%) (predominantly tetralogy of Fallot, complete atrioventricular canal defects, patent ductus arteriosus, atrial and ventricular septal defects, etc.), neurologic (4\%) (central and neuromuscular abnormalities), 


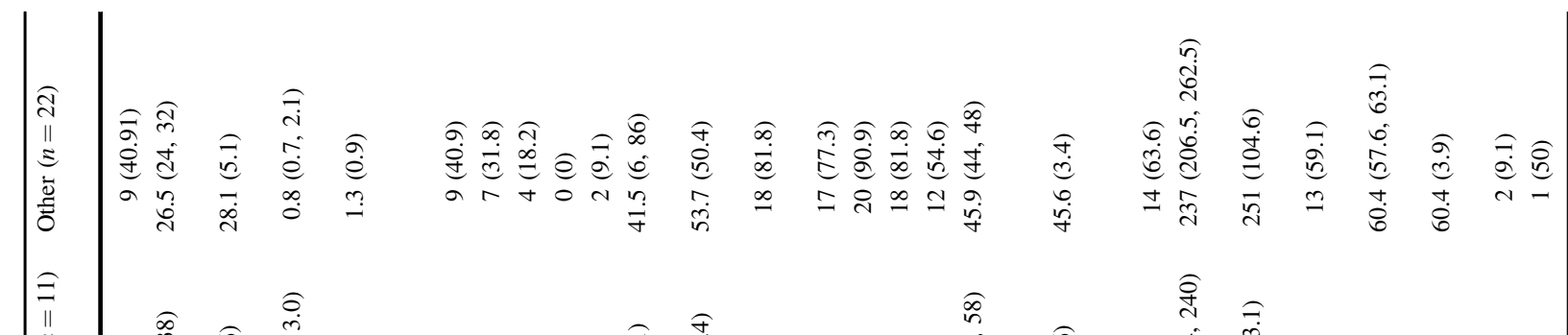

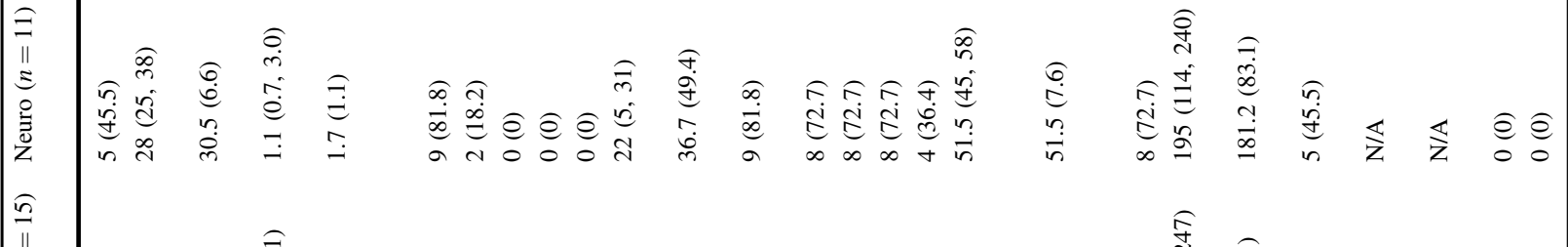

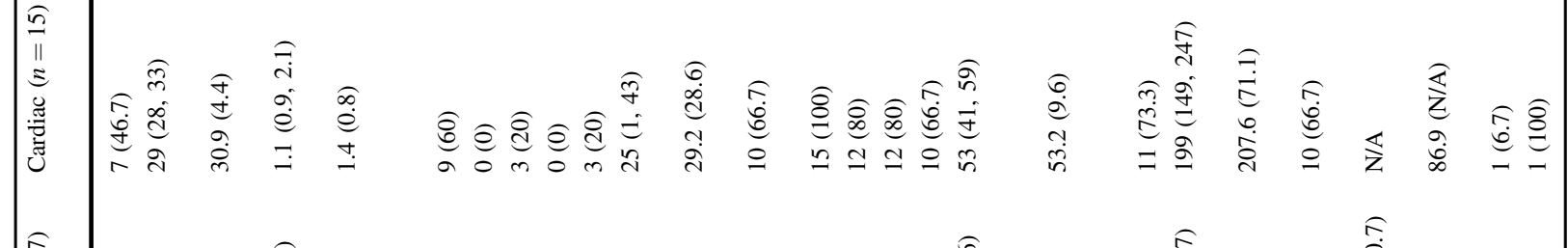

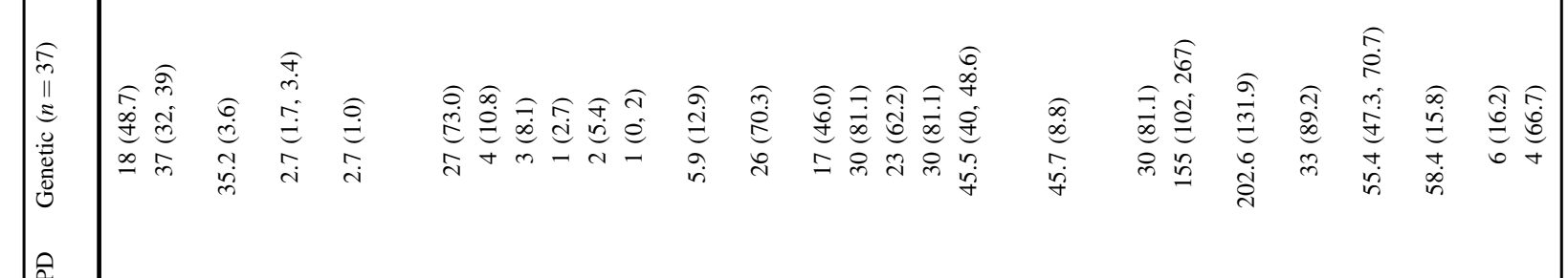

$$
\begin{aligned}
& \text { क्षे }
\end{aligned}
$$

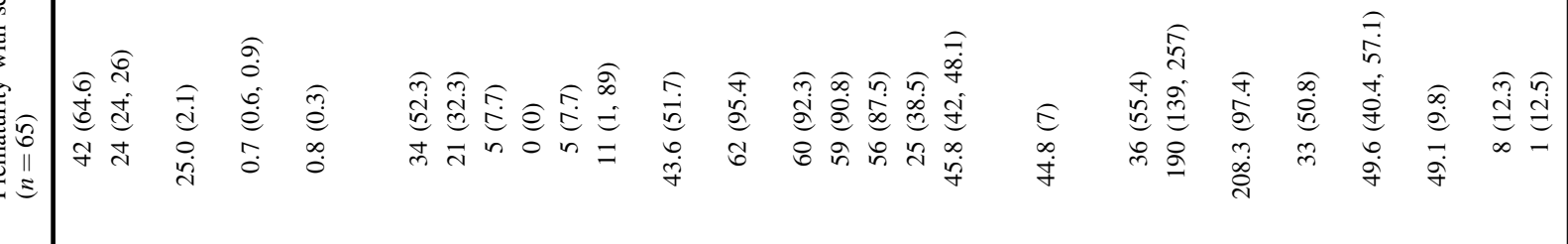

$$
\begin{aligned}
& \text { 命 }
\end{aligned}
$$

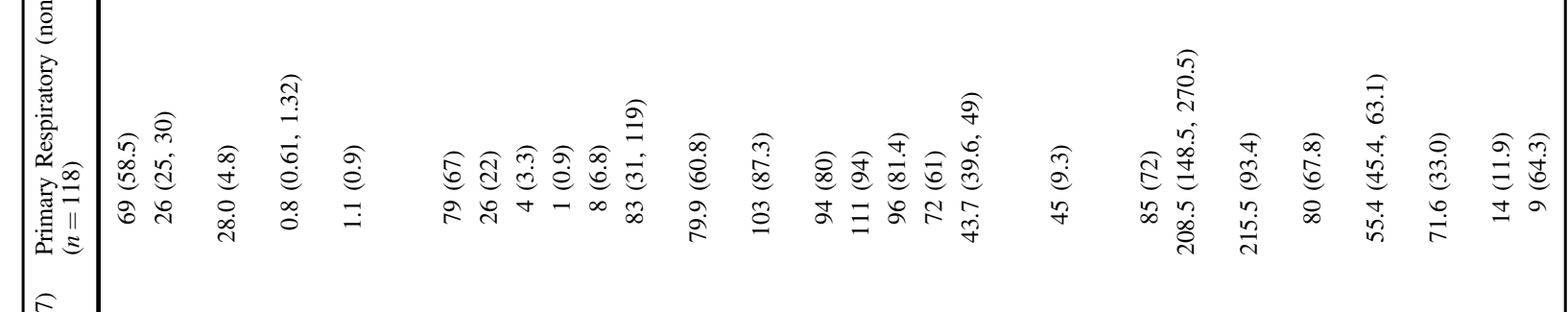

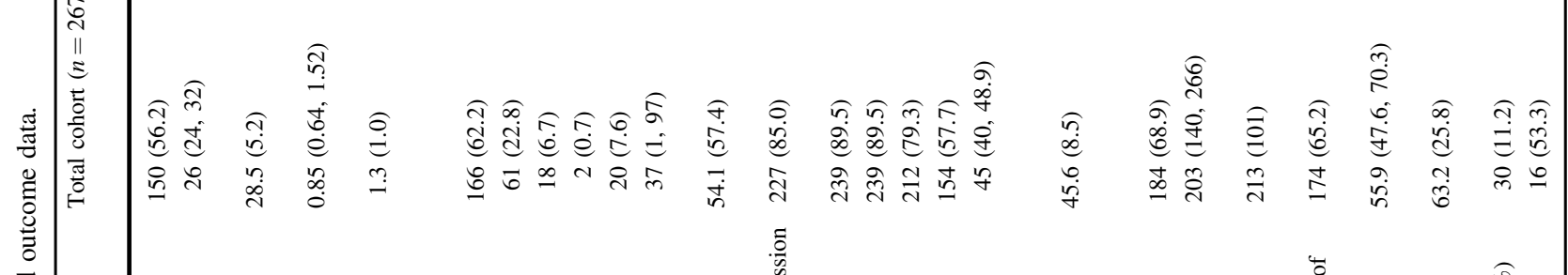

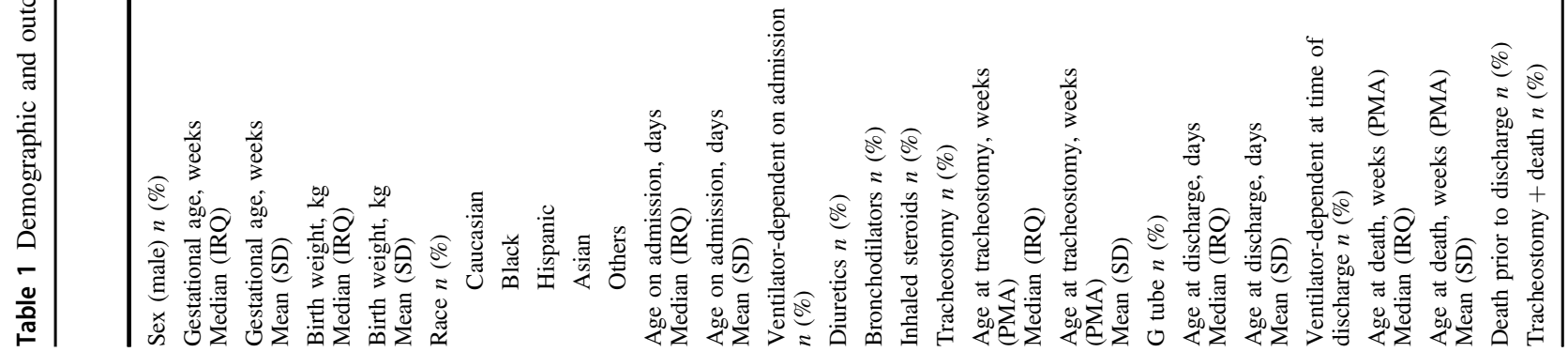


and others (8\%) composed of patients with primary diagnosis other than any of the five subgroups (gastrointestinal diagnosis, feeding intolerance, severe retinopathy of prematurity, etc.) (Table 1).

Patients with primary respiratory diagnosis and those extremely premature at birth were significantly smaller $(0.8 \mathrm{~kg}$ [IQR $0.61,1.32$ ] and $0.7 \mathrm{~kg}$ [IQR $0.6,0.9$ ], respectively). Of the 267 patients, $54.3 \%$ were in-born and $45.7 \%$ were out-born and cared for initially in one of the level-II and-III NICUs in the region. The median age at the time of admission was 37 days (IQR: 1, 97); mean [SD] was 54.1 days [57.4]. The patients with genetic diagnosis were admitted at a much younger age, median age of 1 day (IQR 0,2 ), while patients with primary respiratory (non-BPD) conditions were admitted at much older age, median age of 83 days (IQR 31,119). Many patients in the cohort (85.0\%) were ventilator-dependent at the time of admission. The proportion of ventilator-dependent patients was similar across all the six diagnosis subgroups (Table 1).

Medication usage during hospitalization varied depending on the primary diagnosis. The most-common medication categories were diuretics, systemic steroids, inhaled steroids, bronchodilators, inhaled nitric oxide, and PDE5 inhibitors. Over $75 \%$ of all patients in the cohort received diuretics, systemic and inhaled steroids, and bronchodilators (Table 1). Pulmonary hypertension was diagnosed in $37.2 \%$ of the patient population, $39.7 \%$ were treated with inhaled nitric oxide, and $<18 \%$ required PDE5 inhibitors.

\section{Outcome data}

There was an overall increasing trend in the total number of patients and the number of patients in each of the six subgroup diagnostic categories over time, with a notable significant increase in cases with prematurity with severe BPD and genetic conditions in 2017, as shown in Fig. 1. Of the 267 infants in the study, $154(57.7 \%)$ required tracheostomy, with the highest proportion of tracheostomy performed in patients belonging to the genetic diagnosis group $(81 \%)$. The timing of when tracheostomy was performed was at a median age of 45 (IQR: 40, 48.9) weeks PMA; mean (SD) was 45.6 (8.5) weeks PMA and was consistent regardless of the primary diagnosis subgroup. All patients were ventilator-dependent at the time of tracheostomy, with a mean respiratory severity score (RSS) of $6( \pm 8.89)$. Most patients who received tracheostomy had gastrostomy tubes placed (68.9\%), 13.5\% had Nissen fundoplication (Table 1) and none received transpyloric tube.

A simple linear regression showed that, over time, there was a positive average trend for the number of patients who required tracheostomy, while the mortality rates improved as shown in Fig. 2. Of note, in 2018, there was a decrease in
Fig. 1 Diagnosis trend over time. Prematurity with severe BPD and genetic diagnosis have increased on average over time.

Fig. 2 Simple linear regression (dotted lines) shows a positive average trend for number of patients with tracheostomy over time. Death remained the same.
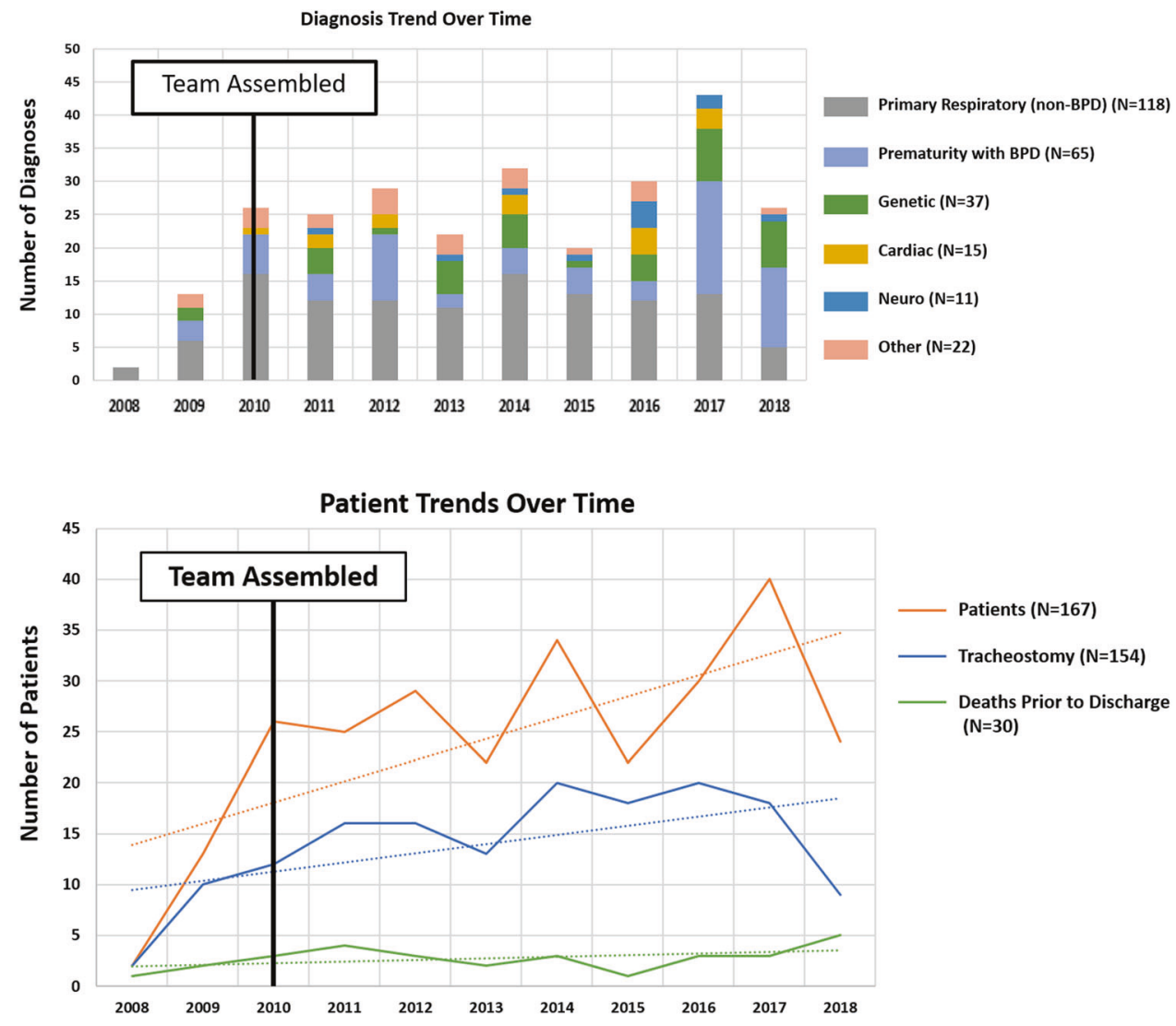
the total number of patients that also corresponded to a decrease in the number of patients who received tracheostomy (Fig. 2).

Unadjusted logistic regression analysis showed that tracheostomy was not associated with the odds of death before discharge. However, secondary pulmonary hypertension as a comorbidity was associated with the odds of combined outcome of death + tracheostomy $(\mathrm{OR}=13.58 \mathrm{CI}$ [3.02, $61.14] ; p$ value $=0.0007)$, or death $(\mathrm{OR}=8.59$ CI [3.37, 21.89]; $p$ value $=<0.0001)$, or tracheostomy $(\mathrm{OR}=1.8 \mathrm{CI}$ $[1.07,3.01] ; p$ value $=0.0264)$ (Table 2). A total of 30 $(11.2 \%)$ patients died prior to discharge with the median age at time of the death of 177 days (IQR: 136, 266) or 55.9 (IQR 47.6, 70.3) weeks PMA; mean (SD) was 237 days (187) or 63.2 (25.8) weeks PMA. The causes of death were confirmed by autopsy in nine patients $(30 \%)$ and the rest were determined by case reviews. The most-common cause of death was irreversible BPD-associated pulmonary hypertension in 11 (37\%) followed by genetic and congenital anomalies in $9(30 \%)$. The overall survival rate to hospital discharge for the entire cohort was $88.8 \%$, with a median age at discharge of 203 days (IQR 140, 266). Sixtyfive percent of patients with tracheostomy were discharged home on assisted ventilation (Table 1).

\section{Discussion}

We describe a consistent coordinated multi- and interdisciplinary approach in the care of medically complex patients with chronic lung disease in a large regional referral ICN and its effect on the survival to discharge of infants with tracheostomy. The ATS defines CLDI as a heterogeneous group of respiratory diseases of infancy that begins in the neonatal period. CLDI most commonly occurs in infants with birth weight $<1000 \mathrm{~g}$ and who were treated for respiratory distress syndrome. However, any neonatal condition that produces an acute lung injury and/or requires positive pressure mechanical ventilation and high concentrations of inspired oxygen during the initial weeks of life predisposes the infant to the development of CLDI. This broad category of diseases includes, most commonly, BPD, a term often used interchangeably with CLD, and other chronic pulmonary conditions in term newborns that result from aspiration syndromes, infections, pulmonary hypoplasia, persistent pulmonary hypertension, congenital diaphragmatic hernia, tracheoesophageal fistula, congenital cardiac disease, congenital neuromuscular disorders, and various genetic conditions with airway and pulmonary abnormalities [5].

A multidisciplinary team approach to the care of infants with severe BPD/CLDI is now becoming the standard of care. The BPD collaborative group has published guidelines to care for infants with severe BPD that include strategies for mechanical ventilation, pulmonary function testing, surveillance for BPD-associated pulmonary hypertension, and nutritional support all the way to post-hospital discharge follow-up care [7]. Since the organization of our dedicated CLD multidisciplinary team in 2010, we have implemented consistent evidence-based consensus-driven guidelines for the care of infants with severe BPD/CLD in our unit. Most of our patients were categorized as having primary pulmonary and airway conditions that evolved into what is broadly considered CLDI, while a quarter of the cohort had the strict definition of severe BPD. Also of note, those infants in the genetic subgroup were more mature at birth with a median gestational age of 37 weeks.

Table 2 Discharge or tracheostomy before discharge.

\begin{tabular}{llll}
\hline Comorbidities & $\begin{array}{l}\text { Death prior to dc }+ \text { trach } \\
\text { outcome OR }[95 \% \text { CI; } p \text { value }]\end{array}$ & $\begin{array}{l}\text { Death prior to dc outcome } \\
\text { OR }[95 \% \text { CI; } p \text { value }]\end{array}$ & Trach outcome OR [95\% CI; $p$ value $]$ \\
\hline PHTN & $13.58[3.02,61.14] ; 0.0007$ & $8.59[3.37,21.89] ;<0.0001$ & $1.80[1.07,3.01] ; 0.0264$ \\
Cardiac & $0.84[0.30,2.32] ; 0.7330$ & $1.36[0.61,3.04] ; 0.4507$ & $0.89[0.54,1.46] ; 0.6375$ \\
Neuro & $0.60[0.20,1.78] ; 0.3596$ & $0.65[0.29,1.45] ; 0.2931$ & $1.27[0.78,2.09] ; 0.3381$ \\
Genetic & $1.67[0.55,4.98] ; 0.3671$ & $1.92[0.84,4.36] ; 0.1202$ & $1.09[0.61,1.96] ; 0.7722$ \\
Ophthalmic & $0.52[0.19,1.45] ; 0.2137$ & $0.58[0.27,1.23] ; 0.1554$ & $0.80[0.49,1.31] ; 0.3714$ \\
Infection & $0.55[0.05,6.59] ; 0.6396$ & $2.17[0.24,19.28] ; 0.4881$ & $2.04[0.56,7.39] ; 0.2795$ \\
BPD & $1.89[0.52,6.84] ; 0.3301$ & $1.18[0.50,2.78] ; 0.6998$ & $0.85[0.49,1.45] ; 0.5387$ \\
GI & $0.66[0.22,1.97] ; 0.4602$ & $0.72[0.32,1.61] ; 0.4255$ & $1.24[0.75,2.03] ; 0.4067$ \\
G tube & $7.28[0.95,56.01] ; 0.0567$ & $0.40[0.19,0.69] ; 0.023$ & $9.28[5.06,17.04] ;<0.0001$ \\
Fundo & $0.91[0.20,4.19] ; 0.9055$ & $0.43[0.10,1.87] ; 0.2591$ & $1.80[0.84,3.82] ; 0.1283$ \\
\hline
\end{tabular}

Logistic regression analysis was completed for ten different comorbidities for outcomes included: death or tracheostomy prior to discharge, and combined outcome of death and tracheostomy prior to discharge. Pulmonary hypertension was associated with the odds of all three outcomes assessed.

$d c$ discharge, PHTN pulmonary hypertension, trach tracheostomy, G tube gastrostomy tube, fundo fundoplication. 
It is not surprising that almost half of our cohort in our regional referral NICU in a free-standing children's hospital were out-born and transferred to our institution at an older age, i.e., at a median age of 37 days (mean of 54 days). However, those infants with genetic diagnosis were admitted to our care, mostly soon after birth, as most of them were born in our Fetal Health Center that provides delivery service to infants with congenital anomalies known prenatally. It is also expected that the majority of the infants remained ventilator-dependent at the time of admission. In fact, most of them were mechanically ventilated since birth, with some failing multiple attempts of extubation, and then transferred to our center for tracheostomy evaluation. Most have been tried on different modes of ventilation at the birth hospitals. Specific to ventilation strategy for severe BPD/ CLD, our team has applied our consensus approach (Appendix 1) that is consistent with the recently published ventilation strategies in severe BPD [8]. In addition, a relatively newer ventilation strategy called neutrally adjusted ventilatory assist mode, both invasively or noninvasively, has been used for our patients with severe BPD, and has been found to be safe and effective [9, 10]. The use of various medications to ameliorate the severity of BPD has been controversial, specifically the use of diuretics, systemic and inhaled steroids, bronchodilators as well as antibiotics. Recently, it was reported that infants with severe BPD are exposed to an alarming number of medications of unclear efficacy and safety, with marked variation between centers $[11,12]$. The role of our clinical pharmacologist as a member of the CLD team cannot be overemphasized, as she plays a critical role in the timing, duration, and rationale for use of such medications. However, even with judicious oversight, over $75 \%$ of our patients received diuretics, steroids, and bronchodilators during their hospitalization. This practice is continuously being monitored and evaluated to decrease medication exposures of these infants. To decrease antibiotic exposure of chronically intubated patients, we implemented our tracheitis algorithm as a QI initiative with the support of our infectious disease experts. This practice has decreased the overuse of antibiotics and has been shared within the NICU and pediatric intensive care unit (Appendix 3).

Over the 9-year period since organizing our CLD team, the number of patients with various pulmonary conditions cared for by the team has increased. While various nonBPD respiratory conditions remain the most-common conditions treated by our team, the biggest increase has been in the number of extremely premature infants with severe BPD and those with a genetic diagnosis. This increase is likely due to multiple reasons, including the overall increase in the survival of extremely premature infants that require longterm ventilatory support [13], the widening catchment area of our referral base (Appendix 5), the earlier recognition by our referring NICUs of the need for tracheostomy for home ventilation, as well as parental choice of continuing lifesustaining assisted respiratory support for infants with complex congenital anomalies.

The decision to perform tracheostomy in infants remains challenging as there are no national standards as to what type of population of infants qualify and what is the optimal timing, and what are the long-term pulmonary outcomes. With advancement in neonatal care and increasing survival of extremely premature infants, most tracheostomies are now being performed in infants $<12$ months of age [14]. The indications for tracheostomy in infants have also shifted from congenital or acquired airway obstruction to preterm infants who developed severe BPD/CLD, neurologic conditions, cardiac anomalies, as well as genetic conditions [15]. In our cohort, $38.5 \%$ of premature infants with severe BPD, and $61 \%$ of infants with other congenital or acquired non-BPD respiratory conditions had tracheostomy performed. However, the largest proportion was among those infants with genetic diagnosis, 30 out of 37 (81\%) received tracheostomy. Most tracheostomies in our cohort were performed primarily for long-term assisted ventilation at home. In the largest multicenter study to date, tracheostomy was performed in only $0.75 \%$ of 458,624 very-low birthweight infants $(<1500 \mathrm{~g})$ born between 2006 and 2016 . However, the number of tracheostomies increased to $5.4 \%$ for infants with birth weight $<1000 \mathrm{~g}$, and $15.8 \%$ in those infants with any congenital malformation. In the same study, CLD and congenital anomalies were the strongest predictors of tracheostomy placement and mortality [15].

The median age at tracheostomy placement in our cohort was 45 weeks PMA, which is younger than what Murthy et al. and Mandy et al. reported, 46 and 51 weeks' PMA, respectively $[16,17]$. However, our data are more consistent with the results of a large multicenter retrospective study from the NICHD Neonatal Research Network that reported that patients with tracheostomy performed at $<120$ days of age had better neurodevelopmental outcomes compared to those with tracheostomy placed after 120 days [18]. A gastrostomy tube is placed almost invariably at the same time of tracheostomy in our cohort to guarantee long-term optimal nutritional support. This dual placement has been a consensus practice specific to our center and may not necessarily be an independent risk factor for tracheostomy placement.

Among the different comorbid conditions that we analyzed, we found that BPD-associated secondary pulmonary hypertension was strongly associated with the need for tracheostomy, or death, or combined outcome of tracheostomy and death. Secondary pulmonary hypertension is a known complication of severe BPD/CLD and contributes to early mortality [19]. The role of prematurity, inflammation, and oxidative stress in the pathophysiology of 
pulmonary hypertension in BPD has been shown to result into deregulation of various signaling pathways leading to impaired alveolarization and disrupted angiogenesis [20]. The reported incidence of BPD-associated pulmonary hypertension varies between 20 and 40\% [21, 22]. We found our rate $(37.2 \%)$ to be at the higher end, likely because we do surveillance echocardiogram in all infants with severe BPD, and we collaborate actively with our pulmonary hypertension team, who are members of our interdisciplinary CLD team. We also consider the presence of worsening BPD-associated pulmonary hypertension as an early indication for tracheostomy placement. Notably, those infants in our cohort with early echocardiographic evidence of pulmonary hypertension and left ventricular dysfunction have a higher risk of developing acute posttracheostomy clinical decompensation [23].

The overall survival rate of our infants with severe CLD cared for by our multidisciplinary team was $88.8 \%$, and $65 \%$ of them were ventilator-dependent through a tracheostomy at the time of NICU discharge. The benefits of a dedicated, consistent, and standardized approach to the care of these medically complex infants cannot be overemphasized. Similar findings among infants with severe BPD with tracheostomy cared for by an interdisciplinary team in another regional center showed significant improvement in their survival [24]. Interestingly, a recent survey done by the Children's Hospital Neonatal Consortium found that among 31 children's hospital NICUs, $41 \%$ had a self-designated multidisciplinary BPD team (unpublished data). The median age at the time of hospital discharge among our survivors was 203 days (IQR 140, 266).

Multiple factors accounted for prolonged hospitalization of our severe CLD infants who were mostly ventilatordependent. Among them include the education and skills training that the family/caregivers must complete and be competent with (Appendix 4), the availability of private duty nurses to support the family at home, especially for those who live outside of the metropolitan area (Appendix 5), and at times, the psychosocial barriers to discharging these infants safely to biological parents.

\section{Limitations}

A limitation of our study lies in the very nature of a retrospective chart review. There were some challenges to separating the primary diagnoses, particularly for those infants who were born extremely premature with BPD versus those who had other congenital or acquired respiratory and airway conditions. We also had limited access to detailed documentation of the early clinical data. Including documentation of ventilatory support and medication exposures, especially for those infants who were transferred relatively late in their clinical course. Last, the various interventions carried out by our multidisciplinary team occurred over a long period of time. The improvement in the primary outcome could be secondary to the learned experience and expertise of the various team members.

\section{Conclusion}

Over time, mortality improved for infants with tracheostomy cared for by a dedicated multidisciplinary BPD/CLD team.

Tracheostomy is not associated with the odds of death prior to NICU discharge. In a cohort of infants with severe BPD/CLD, secondary pulmonary hypertension is strongly associated with the need for a tracheostomy, or death, or tracheostomy and death.

Acknowledgements The authors acknowledge all the members of the Chronic Lung Disease multidisciplinary team for their dedication to our BPD/CLD patients. The authors also thank the Children's Mercy Medical Writing Center for reviewing and editing this manuscript.

Author contributions TPH and SK collected data with supervision from WM, MN provided the history of CLD team and the patient repository. JN-M performed statistics, figures, and tables. TPH, JN-M, and WM wrote the manuscript in consultation with MN, WT and SK.

\section{Compliance with ethical standards}

Conflict of interest The authors declare no competing interests.

Publisher's note Springer Nature remains neutral with regard to jurisdictional claims in published maps and institutional affiliations.

\section{References}

1. Northway WH Jr., Rosan RC, Porter DY. Pulmonary disease following respiratory therapy of hyaline-membrane disease, Bronchopulmonary dysplasia. N Engl J Med. 1967;276:357-68.

2. Doyle LW, Anderson PJ. Long-term outcomes of bronchopulmonary dysplasia. Semin Fetal Neonatal Med. 2009;14:391-5.

3. Jobe AH, Bancalari E. Bronchopulmonary dysplasia. Am J Respir Crit Care Med. 2001;163:1723-9.

4. Ehrenkranz RA, Walsh MC, Vohr BR, Jobe AH, Wright LL, Fanaroff AA, et al. Validation of the National Institutes of Health consensus definition of bronchopulmonary dysplasia. Pediatrics. 2005;116:1353-60.

5. Allen J, Zwerdling R, Ehrenkranz R, Gaultier C, Geggel R, Greenough A, et al. Statement on the care of the child with chronic lung disease of infancy and childhood. Am J Respir Crit Care Med. 2003;168:356-96.

6. Bapat R, Nelin L, Shepherd E, Ryshen G, Elgin A, Bartman T. A multidisciplinary quality improvement effort to reduce bronchopulmonary dysplasia incidence. J Perinatol. 2020;40:681-7.

7. Abman SH, Collaco JM, Shepherd EG, Keszler M, CuecvasGuaman M, Welty SE, et al. Interdisciplinary care of children with severe bronchopulmonary dysplasia. J Pediatr. 2018;197:300-8. 
8. Gibbs K, Jensen E, Alexiou S, Munson D, Zhang H. Ventilation strategies in severe bronchopulmonary dysplasia. NeoReviews. 2020;21:e226-37.

9. Rosterman JL, Pallotto EK, Truog WE, Escobar H, Meinert KA, Holmes A, et al. The impact of neutrally adjusted ventilatory assist mode on respiratory severity score and energy expenditure in infants: a randomized crossover trial. J Perinatol. 2018;38: 59-63.

10. McKinney RL, Keszler M, Truog WE, Norberg M, Sindelar R, Wallstrom L, et al. Multicenter experience with neutrally adjusted ventilator assist in infants with severe bronchopulmonary dysplasia. Am J Perinatol. 2020. https://doi.org/10.1055/s-00401708559.

11. Bamat NA, Kirpalani H, Feudtner C, Jensen EA, Laughon MM, Zhang $\mathrm{H}$, et al. Medication use in infants with severe bronchopulmonary dysplasia admitted to United States children's hospitals. J Perinatol. 2019;39:1291-9.

12. Greenberg JM, Poindexter BB, Shaw PA, Bellamy SL, Keller RL, Moore PE, et al. Respiratory medication use in extremely premature ( $<29$ weeks) infants during initial NICU hospitalization: results from prematurity and respiratory outcomes program. Pediatr Pulmonol. 2020;55:360-8.

13. Overman AE, Liu M, Kurachek SC, Shreve MR, Maynard RC, Mammel MC, Moore BM. Tracheostomy for infants requiring prolonged mechanical ventilation: 10 years' experience. Pediatrics. 2013;131:e1491-6.

14. Lewis CW, Carron JD, Perkins JA, Sie KCY, Feudner C. Tracheostomy in pediatric patients: a national perspective. Arch Otolaryngol Head Neck Surg. 2003;129:523-9.

15. Han SM, Watters KF, Hong CR, Edwards EM, Knell J, Morrow $\mathrm{KA}$, et al. Tracheostomy in very low birth weight infants: a prospective multicenter study. Pediatrics. 2020;145:1-9.
16. Murthy K, Porta NFM, Lagatta JM, Zaniletti I, Truog W, Grover $\mathrm{T}$, et al. Inter-center variation in death or tracheostomy placement in infants with severe bronchopulmonary dysplasia. J Perinatol. 2017;37:723-7.

17. Mandy G, Malkar M, Welty SE, Brown R, Shephard E, Gardner $\mathrm{W}$, et al. Tracheostomy placement in infants with bronchopulmonary dysplasia: safety and outcomes. Pediatr Pulmonol. 2013;48:245-9.

18. Demauro SB, D’Agostino JA, Bann C, Bernbaum J, Gerdes M, Bell E, et al. Developmental outcomes of very preterm infants with tracheostomies. J Pediatr. 2014;164:1303-10.

19. Khemani E, McElhinney DB, Rhein L, Andrade O, Lacro R, Thomas K, et al. Pulmonary artery hypertension in formerly premature infants with bronchopulmonary dysplasia: clinical features and outcomes in the surfactant era. Pediatrics. 2007;120:1260-9.

20. Mathew R. Signaling pathways in the development of bronchopulmonary dysplasia and pulmonary hypertension. Children. 2020;7:100. https://doi.org/10.3390/children7080100.

21. Dh Kim, Kim HS, Choi CW, Kim EK, Kim B, Choi JH. Risk factors for pulmonary artery hypertension in preterm infants with moderate to severe bronchopulmonary dysplasia. Neonatology. 2012;101:40.

22. Farquhar M, Fitzgerald DA. Pulmonary hypertension in chronic neonatal lung disease. Pediatr Respir Rev. 2010;11:149.

23. Nyp MF, Taylor JB, Petralia A, Oschman A, Norberg M, Weatherly $\mathrm{R}$, et al. Acute post-tracheostomy clinical decompensation in infants-are there predictive markers? Am J Perinatol. 2018;35:1206-12.

24. Gien J, Kinsella J, Thrasher J, Grenolds A, Abman S, Baker C. Retrospective analysis of an interdisciplinary ventilator care program intervention on survival of infants with ventilator-dependent bronchopulmonary dysplasia. Am J Perinatol. 2017;34:155-63. 